\title{
Spatial temporal cluster analysis to enhance awareness of disease re-emergence on a global scale
}

\author{
Forest M. Altherr ${ }^{\star 1}$, Maneesha Chitanvis ${ }^{1}$, Ashlynn Daughton ${ }^{1,2}$, Geoffrey Fairchild1, \\ William Rosenberger ${ }^{1}$, Nicholas Generous ${ }^{1}$, Nidhi Parikh${ }^{1}$, Derek Aberle ${ }^{1}$, \\ Nileena Velappan ${ }^{1}$, Emily Alipio Lyon ${ }^{1}$, Attelia Hollander ${ }^{1}$ and Alina Deshpande ${ }^{1}$
}

${ }^{1}$ Bioscience Division, Los Alamos National Laboratory, Los Alamos, NM, USA; ${ }^{2}$ University of Colorado, Boulder, Boulder, CO, USA

\section{Objective}

The application of spatial analysis to improve the awareness and use of surveillance data.

\section{Introduction}

The re-emergence of an infectious disease is dependent on social, political, behavioral, and disease-specific factors. Global disease surveillance is a requisite of early detection that facilitates coordinated interventions to these events. Novel informatics tools developed from publicly available data are constantly evolving with the incorporation of new data streams. Re-emerging Infectious Disease (RED) Alert is an open-source tool designed to help analysts develop a contextual framework when planning for future events, given what has occurred in the past. Geospatial methods assist researchers in making informed decisions by incorporating the power of place to better explain the relationships between variables.

\section{Methods}

Disease incidence and indicator data derived for the RED Alert project were analyzed for spatial associations. Using aggregate country-level data, spatial and spatiotemporal clusters were identified in ArcMap 10.5.1. The identified clusters were then used as the outcome for a series of binary logistic regression models to determine significant covariates that help explain global hotspots. These methods will continue to evolve and be incorporated into the RED Alert decision support ecosystem to provide analysts with a global perspective on potential re-emergence.

\section{Results}

Hotspots of high disease incidence in relation to neighboring countries were identified for measles, cholera, dengue, and yellow fever between 2000 and 2014. Disease-specific predictors were identified using aggregate estimates from World Bank indicator dataset. Data was imputed where possible to enhance the validity of the $\mathrm{Gi} *$ statistic for clustering. In the future, as data streams become more readily available, hotspot modeling at a finer resolution will help to improve the precision of spatial epidemiology.

\section{Conclusions}

Spatial methods enhance the capability of understanding complex population and disease relationships, which in turn improves surveillance and the ability to predict re-emergence. With tools like RED Alert, public health analysts can better prepare to respond rapidly to future re-emerging disease threats.

\section{Keywords}

Re-emergence; Surveillance; Spatial; Hotspot

\section{${ }^{\star}$ Forest M. Altherr}

E-mail: faltherr@lanl.gov 